\title{
The Extreme Ultraviolet Explorer Public Right Angle Program
}

\author{
K. McDONALD, N. CRAIG, E. OLSON, AND C. A. CHRISTIAN \\ Center for EUV Astrophysics, 2150 Kittredge St., University of California, \\ Berkeley, CA 94720-5030, USA
}

The new Extreme Ultraviolet Explorer (EUVE) Public Right Angle Program (RAP) offers the opportunity for researchers to obtain observations with the $E U V E$ imaging telescopes, oriented at right angles to the EUVE spectrometer used for the NASA Guest Observer Program. Scientists may submit proposals electronically through the World Wide Web or e-mail using a template form to list specific targets and present the scientific motivation for the work. The RAP electronic proposal process is streamlined from proposal submission through data delivery and is a prototype for a system to be used for all guest observers during the EUVE extended mission starting in February, 1996.

\section{Introduction}

The Extreme Ultraviolet Explorer (EUVE) Public Right Angle Program (RAP) is a new program that makes the observations taken with the EUVE scanning telescopes available to scientists around the world. These imaging instruments, primarily used during the EUVE all-sky survey (Bowyer et al. 1994; Bowyer et al. 1996), are pointed along a great circle oriented perpendicular to the EUVE Deep Survey/Spectrometer used for guest observer observations. The scanners can be used to collect data of high scientific interest simultaneously with the spectroscopy of scheduled GO targets. The RAP was started at CEA as a way to optimize the data collected by specifying particular spacecraft roll angles (subject to spacecraft and instrument pointing constraints) so that targets of astrophysical significance could be observed (McDonald et al. 1994). The goal of the Public RAP is to provide a simple, effective, on-line method to propose targets, schedule observations, and disseminate data products to the scientific community as well as provide a testbed for the EUVE extended mission GO program.

\section{Proposal Submission Process}

RAP proposals are submitted by accessing and completing the proposal form available through the WWW. The ASCII format form is sent via electronic mail and processed through the EUVE Project Office at Goddard Space Flight Center. Approved targets are added to the database of targets available for scheduling. If a target satisfies the pointing constraints, it is placed in the upcoming EUVE schedule, and the Principal Investigator is notified by electronic means.

\section{Data Reduction and Delivery for RAP Targets}

When a scheduled RAP target has been observed, the data is reduced using the standard EUV package software in IRAF and FITS formats which preserve the event data. These data are distributed to the Principal Investigator via any of a number of different media (ftp, $8 \mathrm{~mm}$ tar, CD-ROM, etc.). The Principal Investigator is awarded 6 months proprietary data rights to the data taken during this observation, at which time the data 
will become public domain and accessible to anyone upon requests. Information about observed targets are published in the EUVE Electronic Newsletter on a monthly basis. No financial support is provided to investigators for analysis of this data via the Public RAP, but all investigators are encouraged to apply for other means of financial support (such as NASAs Astrophysics Data Program).

\section{Application of Public RAP to EUVE Extended Mission}

In February 1996, the EUVE satellite will enter the extended mission phase in which changes to science operations will be implemented. Procedures to streamline all parts of the system, including observing proposals, observation scheduling, data delivery are being tested currently. Since the Public RAP has similar characteristics to those planned for the EUVE extended mission, the proposal system of the RAP serves as a working model for the methods to be used throughout the EUVE and other NASA satellites.

This work has been supported by NASA contract NAS5-29298.

\section{REFERENCES}

Bowyer, S., Lieu, R., Lampton, M., Lewis, J., Wu, X., Drake, J. J., \& Malina, R. F. 1994, The First Extreme Ultraviolet Explorer Source Catalog, ApJS, 93, 569

Bowyer, S. \& Malina, R. F. 1991, The Extreme Ultraviolet Explorer Mission, in Extreme Ultraviolet Astronomy, ed. R. F. Malina \& S. Bowyer, New York: Pergamon Press, 387

BOWYER, S. ET AL. 1996, ApJS, in press

McDonald, K., Craig, N., Sirk, M. M., Drake, J. J., Fruscione, A., Vallerga, J. V., \& Malina, R. F. 1994, Serendipitous EUV Sources Detected During the First Year of the Extreme Ultraviolet Explorer Right Angle Program, AJ, 108, 1843 